4 tis

TRANS $\cdot \mathrm{N} .5 \cdot 200 \mathrm{I}$

ARTICULOS $\cdot 103-110$
El articulo parte de la importancia que la comunicación no verbal tiene sobre la interpretación, en concreto se estudian los efectos que la entonación monótona pueda tener sobre la recuperación de la información en la interpretación simultánea. $\mathrm{El}$ análisis se basa en un estudio experimental realizado por la autora. Como material se utilizaron tres videos en los que se manipuló la entonación de la interpretación y que contenian tanto la exposición o ponencia original como las interpretaciones superpuestas. Los sujetos, 42 profesores de las Facultades de Derecho y Ciencias Politicas y Sociologia de la Universidad de Granada, asi como 15 intérpretes profesionales, fueron repartidos en tres subgrupos. Todos los sujetos vieron y contestaron a sendos cuestionarios sobre los videos. Los resultados confirman los efectos negativos de la entonación monótona sobre la recuperación de la información.

\title{
Efectos de la entonación monótona sobre la recuperación de la información en receptores de interpretación simultánea
}

Ángela Collados Ais Universidad de Granada
This article is based on the important role that non-verbal communication plays in conference interpretation. More specifically, it studies the effect that de monotonous intonation can have on the recuperation of information in simultaneous interpretation. The analysis described is based on an experimental study carried out by the author. The materials used were three video recordings of the same interpreted discourse in which the intonation of the interpretations was manipulated. The subjects, 42 orofessors form the Faculties of Law and Political Science and Sociology of the University of Granada as well as 15 professional interpreters, were divided into three subgroups. All of the subjects viewed the videos and afterwards answered questionnoires regarding their contents. The results confirm the negative effects of monotonous intonation in the recuperation of information. 


\section{PLANTEAMIENTO GENERAL}

Partimos de la base de que la voz, en general la conducta vocal del intérprete no ha gozado hasta ahora de los favores de los investigadores, entre otras cosas, porque la investigación, joven todavía en el ámbito de la interpretación de conferencias, ha concentrado sus esfuerzos en temas aparentemente de mucha más trascendencia (v.gr. la transmisión de la información) o simplemente más urgentes para poder reafirmarse como disciplina (v. gr. el proceso de la interpretación). Sin embargo, opinamos que actualmente la investigación ya puede comenzar, $y$ de hecho ha comenzado, a diversificarse $y$ que ha llegado la hora de dedicarnos a otros temas que no dejan de ser, como mínimo, igual de importantes que los anteriores.

En este sentido, la relevancia de la conducta vocal del intérprete es manifiesta si tenemos en cuenta que éste comunica un contenido verbal, siguiendo un código lingüístico, pero también comunica siguiendo un código no verbal. Y es precisamente este último ámbito el que, si cabe, ha sido todavía más descuidado. Existe un número considerable de comentarios genéricos sobre la importancia de la voz del intérprete, en el sentido de que ésta no debe ser desagradable ni monótona (Katz, I989) y sí debiera ser agradable porque así el intérprete casi siempre llevaria varios puntos de ventaja (Herbert, 1970). Sin embargo, estos comentarios comparados con la importancia otorgada a otros temas, pueden parecer absolutamente reducidos. No debe extrañar pues que en los estudios de interpretación, en el ámbito de la didáctica, su tratamiento esté también a la zaga de otros parámetros de calidad cuya importancia sí es reconocida unánimemente (v.gr. transmisión correcta del sentido). Su inclusión en el cajón de sastre de otras cuestiones a tener en cuenta por el intérprete condena a la expresión vocal no verbal a su marginalización.

Un intento para integrar el ámbito no verbal y específicamente el no verbal vocal en la interpretación simultánea (IS) ha sido llevado a cabo por Pöchhacker (1994). Pöchhacker integra tanto la vertiente vocal como expresamente la no verbal vocal, otorgándole así un rango de importancia que es el que efectivamente adquiere en IS, donde toda la conducta no verbal debe ser transmitida a través de la voz. Pero la práctica no existencia de estudios específicos sobre la voz en interpretación confirma que ésta es la gran descuidada, como en general toda la comunicación no verbal (CNV) en los estudios de interpretación. Al día de hoy, únicamente pueden ser resaltados tres trabajos específicos sobre la voz del intérprete. Así, Daró (I990) estudia, a través de la Frecuencia fundamental (Fo) las diferencia de tono entre distintas lenguas de trabajo de una intérprete de conferencias concluyendo que existen diferencias importantes según la vinculación del intérprete con la lengua. La Fo es uno de los parámetros vocales más importantes, siendo admitida unánimemente su implicación emocional. Otro de los trabajos que han seleccionado la voz como objeto de estudio es la investigación de Shlesinger (I994) sobre el nivel de retención en IS. Su trabajo muestra que la retención a partir de una IS, en general, es inferior al grado de retención que se alcanza a partir de textos leídos. Shlesinger comparó la emisión vocal de la IS con la lectura, concluyendo que existían rasgos peculiares propios de la entonación del intérprete y, en general, en la emisión vocal de los mismos que se centran fundamentalmente en un incremento importante de pausas no gramaticales, en posición «no usuales», y en una prosodia específica. Por último, Collados (1998), partiendo de la diferenciación entre calidad y éxito, así como de expectativas de calidad y evaluación, investiga la incidencia de la entonación monótona sobre la evaluación de los receptores de IS y lo compara con la incidencia del parámetro de la transmisión correcta del sentido, concluyendo que la entonación monótona del intérprete incide muy negativamente sobre la evaluación de la calidad de la IS efectuada por los receptores. 
No obstante todo lo anterior, el descuido de la voz del intérprete como objeto de estudio no es algo privativo de la interpretación, sino que tiene correlación con los estudios, en general, sobre expresión vocal. Estos se han situado fundamentalmente en la expresión vocal verbal, más que en la expresión vocal no verbal. Los motivos, además de los aducidos en el párrafo anterior, podrían ser, por tanto, considerados no específicos de la interpretación. Así, el hecho de que la conducta vocal no verbal, no únicamente en interpretación, se quedara descolgada de los estudios sobre conducta vocal verbal (Fernández Dols et al., 1990), por las propias dificultades que entraña su estudio, entre otras, por la gran variabilidad intra- $\mathrm{e}$ interindividual y la dificultad de proceder a una separación limpia entre los códigos verbales y no verbales (Kramer, r963).

Sin embargo, es precisamente a partir y a través del canal vocal no verbal por el que emitimos un número de mensajes paralelos, en ocasiones, superiores en cuanto a importancia al de la comunicación verbal (Knapp, 1988). A partir de estudios psicológicos se ha demostrado cómo la monotonía de la voz puede afectar negativamente a la comprensión, la persuasión (Mehrabian \& Williams, I969) y a la percepción (Mahl \& Schulze, I982; Scherer, I995). Según Knapp:

«Es probable que el carácter monótono y desagradable de la voz afecten la comprensión, pero aún así, el oyente puede adaptarse hasta tal punto que retenga una parte considerable de la información comunicada. Probablemente, la pobreza de las cualidades vocales contribuya más a la percepción de la personalidad o al talante del hablante por parte del oyente, que a la disminución de la comprensión» (Knapp, ig88: 306).

Aunque, según nuestro punto de vista, a pesar de una clara interacción, hay diferencias entre comprensión y retención: se puede com- prender algo y no retener. De hecho pudiera ser que la retención, a partir del carácter monótono de la voz, pudiera ser afectada no tanto por la comprensión como por la atención, que se vería notablemente mermada, no llegando el receptor, al menos en determinados pasajes del discurso recibido, a la fase de comprensión.

El trabajo que aqui presentamos se referirá a la retención, no a la comprensión, que al igual que la atención podria situarse en la vía por la que se produciría esa previsible merma de retención. Por tanto, la investigación intenta estudiar, en la parcela que analiza, la importancia que para la IS tiene la comunicación no verbal, teniendo en cuenta que la voz del intérprete de simultánea es el único vehículo que éste tiene a su disposición para la transmisión verbal y no verbal del discurso, y siendo la voz, por tanto, la que asume toda la responsabilidad de una interpretación de "calidad» $y / o$ de «éxito" (Collados Aís, 1998). Para ello hemos analizado la incidencia que tiene la entonación monótona en IS, entendida como ausencia o reducción de inflexiones movidas y variadas o, dicho de otra forma, como uniformidad de sus inflexiones (Tomás y Navarro, I974), sobre la recuperación de la información en un grupo de sujetos expertos y en un grupo de intérpretes profesionales que previamente visionaron y escucharon una interpretación simultánea.

El método elegido ha sido el experimental y nuestra hipótesis de partida era, tal y como ha quedado apuntado anteriormente, que la entonación monótona afectaría negativamente a la retención de la información en IS. Se manipularon las variables de entonación y transmisión correcta del sentido y las prestaciones se presentaron mediante grabaciones en vídeo a los sujetos'.

1 El experimento que presentamos a continuación se inserta dentro de una investigación más amplia que estudia fundamentalmente los efectos de la entonación monótonit sobre la evaluación de la calidad por parte de los receptores de la interpretación simultánea (Collados Aís, 1998). Su presentación aquí desbordaría los límites de este articulo. 


\section{MÉTODO}

\section{I Sujetos}

Los sujetos han sido, por una parte, 42 juristas profesores de las Facultades de Derecho y Ciencias Políticas y Sociología de la Universidad de Granada. Todos los sujetos provienen del ámbito del derecho público, en concreto de los Departamentos de Derecho Internacional Público, Derecho Constitucional, Filosofía del Derecho y Ciencia Política. La elección de estas áreas de conocimiento estuvo motivada por la selección del discurso base sobre el que versaría la interpretación (legislación sobre la financiación de los partidos políticos en Alemania). Por otra parte, el segundo grupo de sujetos estaba integrado por 15 intérpretes profesionales residentes en Granada con lengua A/B español. Los sujetos de ambos grupos (expertos e intérpretes) fueron divididos aleatoriamente en tres subgrupos (véase 2.3.).

\subsection{Material \\ 2.2.I Vídeos}

En total se confeccionaron tres vídeos. En primer lugar se efectuó la grabación (Servicio de Video de la Universidad de Granada) del discurso original sobre legislación en materia de financiación de partidos políticos sobre la base de un discurso original del constitucionalista alemán Dr. Hans Peter Schneider. El discurso fue emitido por un profesor alemán, D. Ludwig Schwarz, director del Instituto Alemán en Granada, respetándose en la grabación las convenciones usuales para este tipo de acontecimientos (sala, vestuario ...). A partir de este discurso base, se superpuso la voz de la intérprete, $D^{\text {a }}$ Luisa von Bernstorff, con una interpretación hacia el español realizada según un estudio previo en el que se comprobaron qué estructuras sintácticas mantenían o no los intérpretes del discurso original. La intérprete era una profesional titulada (en aquel momento, 1996 , intérprete de la Comisión de la Unión Europea) que, como tal, tuvo en cuenta incluso la entonación sui generis de los intérpretes (Shlesinger, 1994).

La elección del video como modo de presentación de las interpretaciones estuvo motivada por la necesidad de mantener invariables las mismas condiciones experimentales para los distintos componentes de los grupos de sujetos. Hubiese sido imposible para la intérprete y el ponente, entre otras condiciones, mantener el mismo grado de monotonía en sesiones sucesivas para sujetos distintos y ello hubiese invalidado los resultados.

El resultado fueron tres videos de una duración de diez minutos:

- version $I$ : interpretación monótona sin fallos de contenido (Collados, 2000 a),

- versión 2: interpretación entonativa con fallos de contenido y (Collados, 200ob),

- versión z: interpretación entonativa sin fallos de contenido (Collados, 2000c).

Las interpretaciones de los tres vídeos fueron sometidas a distintos estudios previos que tenían entre sus objetivos el determinar el grado de monotonía de la voz del ponente y de la intérprete. En concreto, se trata de tres estudios, dos de ellos a través del procedimiento de jueces (6o estudiantes de quinto curso de psicología y 72 estudiantes de traducción e interpretación de tercer curso) y el tercero mediante el estudio acústico por ordenador (Visi-Pitch, accesorio del equipo CSL-50, Computarized Speech Lab. Kay-Elemetric) previa digitalización de las muestras de voz correspondientes a cada uno de los vídeos, a fin de determinar la Fo como parámetro fundamental de la entonación. Estos estudios previos permitieron la determinación del grado de monotonía de las interpretaciones y su clasificación tal y como la hemos presentado en líneas precedentes.

\subsubsection{Cuestionario}

El cuestionario que al debian responder los sujetos tras ver el vídeo correspondiente incluye trece preguntas con tres posibles respuestas, 
salvo una pregunta abierta, sobre el contenido del discurso recibido a través de la interpretación. Las preguntas hacen referencia a todo el discurso y se distribuyen a lo largo del mismo. Se intentó que las respuestas pudiesen ser lo más concretas posibles, de forma que no cupiese la interpretación (en sentido hermenéutico) sobre las cuestiones señaladas. El cuestionario fue redactado en forma de elección múltiple, salvo la pregunta que los sujetos debían responder de forma escrita, siendo el mismo para los tres grupos de sujetos. Las diferencias de contenido de las versiones i y 3 , por una parte (idéntico contenido), y 2, por otra, se tienen en cuenta a la hora de la corrección de los cuestionarios (distintas respuestas válidas para uno y otros).

\subsection{Situación experimental}

La manipulación controlada de las variables entonación y transmisión del sentido del discurso original por las que habiamos optado para alcanzar los objetivos marcados podía realizarse de varias formas:

a) Todos los sujetos seleccionados, tanto usuarios como intérpretes, visionarían las tres versiones de IS y responderían a tres cuestionarios.

b) Los sujetos seleccionados serían divididos en tantos subgrupos como versiones de IS había.

Optamos por la división en tres subgrupos a fin de que cada subgrupo, integrado por el mismo número de sujetos y con participación homogénea de los distintos departamentos, en el caso de los usuarios, escuchara y contestara a un cuestionario. El haber optado por que todo el grupo escuchara las tres versiones hubiese supuesto a nuestro modo de ver un alejamiento importante de la realidad puesto que éste no es el caso usual, una distorsión de los resultados para las dos últimas versiones de IS y la posibilidad de que hubiesen participado menos sujetos en los experimentos, ya que se hubiese tri- plicado el tiempo requerido para los mismos y tanto en el caso de los usuarios como en el de los intérpretes, se trata de profesionales altamente cualificados y con escaso tiempo disponible. Los tres subgrupos quedaron finalmente integrados cada uno de ellos por I4 juristas y 5 intérpretes.

Después de visionar el vídeo correspondiente $y$ de cumplimentar un cuestionario sobre evaluación de la calidad en interpretación simultánea se les pidió a los sujetos que cumplimentaran este cuestionario (2.2.2.). Los sujetos desconocían al comienzo del experimento que deberían cumplimentar un cuestionario sobre el contenido de la interpretación para que ningún sujeto intentara retener información artificialmente.

\section{RESULTADOS}

Para permitir la aplicación del método inferencial, en concreto el test no paramétrico de Kruskal y Wallis, se procedió a agrupar el número de aciertos en dos grupos: entre o y 6 , por una parte, y entre 7 y 13, por otra. Cabe, no obstante, reseñar que el riesgo de que se produzca un error de tipo i es mayor en los tests no paramétricos aplicados a muestras reducidas (Siegel \& Castellán, I988).

\section{I Resultados del grupo de sujetos juristas}

Tal y como puede verse en la figura I., existen diferencias importantes en la tasa de recuperación de la información entre los tres vídeos. En el vídeo I, el $64,28 \%$ de los sujetos tuvo un número de aciertos entre o y 6, y el $35,7 \%$ restante entre 7 y 13 . En el vídeo 2 , el $28,57 \%$ de los sujetos consiguió un número de aciertos entre o y 6 , y el $71,42 \%$ entre 7 y 13 . En el video 3 , el $42,85 \%$ de los sujetos obtuvo entre o y 6 aciertos y el $57,14 \%$ los obtuvo de 7 a 13 . A pesar de que las diferencias son notables en los resultados de los grupos, no se alcanzó significación estadística. 


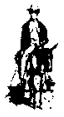

108

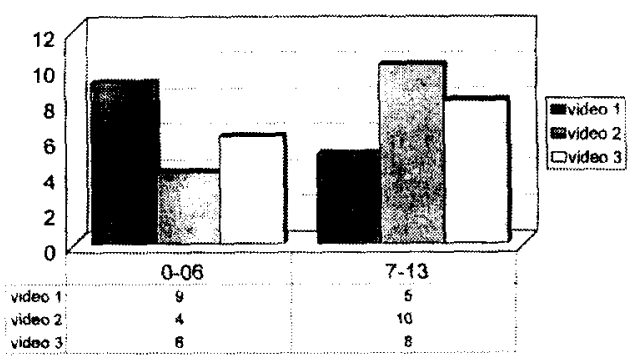

Fig.r. Resultados de respuestas correctas obtenidas por el grupo de juristas

\subsection{Resultados del grupo de sujetos intérpretes}

En la figura 2 podemos apreciar nuevamente las diferencias en la tasa de recuperación de los tres vídeos. En el vídeo I, el $80 \%$ contestó adecuadamente a entre o y 6 preguntas y el $20 \%$ entre 7 y 13 . En el vídeo 2, el $40 \%$ contestó entre o y 6 preguntas y el $60 \%$ entre 7 y I3. En el vídeo 3 , el $60 \%$ contestó entre o y 6 y el $40 \%$ entre 7 y I3. A pesar de las diferencias, éstas no resultaron significativas.

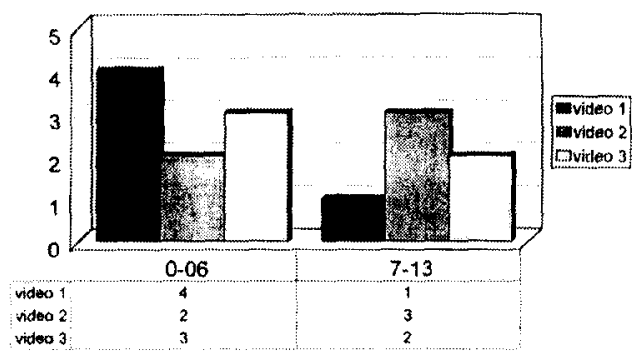

Fig.2. Resultados de respuestas correctas obtenidas por el grupo de intérpretes

\section{DISCUSIÓN}

Es de destacar que en los vídeos 2 y 3 (entonativos), el número de aciertos es claramente superior al del vídeo I (monótono) en todos los casos. Esto nos permite afirmar que, aunque los resultados no fueron significativos, sí existe una clara tendencia a un mayor indice de recuperación de la información cuando la interpretación no ha sido monótona. Dado que el número de sujetos no fue excesivamente alto, es posible que esta tendencia pudiese ser confirmada con un grupo lo suficientemente amplio (véase 3.).

Teniendo en cuenta además que el tiempo que los sujetos de los grupos (juristas e intérpretes) que tuvieron que visionar la versión monótona fue reducido (ro minutos), cabe aventurar la hipótesis (por confirmar) que a mayor tiempo de «exposición» a una interpretación monótona, mayores serían las diferencias en el índice de recuperación entre una interpretación monótona y una interpretación no monótona.

Por otra parte, otra hipótesis que podría lanzarse es que las versiones entonativas contemplan una determinada entonación y que podría suceder que una mayor entonación (límite por determinar) podría causar el efecto de U-invertida $y$, por tanto, invertir sus efectos sobre la recuperación de la información.

Los resultados obtenidos son coherentes con el hecho de que los juristas sean especialistas en la materia objeto del discurso, al haber conseguido un indice de recuperación mayor que los intérpretes en cualquiera de las versiones, tanto entonativas como monótona.

\section{CONCLUSIONES}

La conclusión más importante que podemos extraer de la investigación que presentamos es que efectivamente existe una influencia negativa de la entonación monótona sobre la recuperación de la información por parte de los usuarios analizados.

Abogamos, a partir de esta conclusión, porque el intérprete como comunicador profesional transmita el discurso original de forma que respete la emisión integral del mismo, pero que 
incluso mejore la presentación del emisor original, que no necesariamente tiene que ser también comunicador profesional. Esta defensa del intérprete integral en calidad de comunicador profesional se basa en la consideración inicial del papel del intérprete así considerado y en la convicción de que los usuarios, aunque no lo manifiesten expresamente a priori en sus expectativas, utilizan posteriormente en su evaluación, en este caso en su nivel de retención, $a$ posteriori elementos de los que ellos mismos no tienen porqué ser conscientes.

Los resultados obtenidos permiten aventurar que posiblemente, si se realizasen estudios específicos sobre la conducta vocal del intérprete, podría demostrarse que ésta ejerce influencia, en sus distintos parámetros, sobre más aspectos que el analizado.

Los efectos de ésta y otras investigaciones sobre la voz del intérprete deberían reflejarse en la mayor atención que el intérprete debe prestar a la "forma» en la interpretación y por ende, en el entrenamiento consciente al que debe ser sometido el futuro profesional de la interpretación por el docente de la interpretación. Para ello, la grabación continua de las interpretaciones es un elemento imprescindible, así como la inclusión sistemática de este parámetro en la valoración y calificaciones de las mismas.

\section{RECIBIDO EN SEPTIEMBRE DE 2000}

\section{BIBLIOGRAFIA}

Collados Ais, A. (1998). La evaluación de la calidad en interpretación simultanea. La importancia de la comunicación no verbal. Granada: Comares.

Collados Aís, A. (2000 a). "Interpretación de conferencias: parámetros de calidad V». En: Pamies, A.; Wanning, F. (eds.). Video Tempus Language Toolbox (V'TLT). Hannover/Granada: Método Ediciones\&European Training Foundation (ETF). Gra-912-2000
Collados Aís, A. (200ob). «Interpretación de conferencias: parámetros de calidad IX». En: Pamies, A.; Wanning, F. (eds.). Video Tempus Language Toolbox (VTLT). Hannover/Granada: Método Ediciones \& European Training Foundation (ETF). Gra-912-2000.

Collados Aís, A. (2000c). «Interpretación de conferencias: evaluación de la calidad». En: Pamies, A.; Wanning, F. (eds.). Video Tempus Language Toolbox (VTLT). Hannover/Granada: Método Ediciones \& European Training Foundation (ETF). Gra-912-2000

Daró, V. (I990). «Voice frequency in languages and simultaneous interpretation", The Interpreters' Newsletter 3: 88-92.

Fernández Dols, J.M. et al. (1990). «Comportamiento no verbal y emoción". En: Palafox, S., Vila, J. (eds). Motivación y Emoción. Madrid: Alhambra Universidad, 255-307.

Gile, D. (1995). Regards sur la recherche en interprétation de conférence. Lille: Presses Universitaires.

Herbert, J. (I970). Manual del intérprete. Gincbra: Librairie de l'Université Georg.

Katz, J.D. (I989). «Pour un enseignement de lexpression orale dans les écoles d'Interprètesn. En: Gran, L., Dodds, J. (eds.). The Theoretical and Practical Aspects of Teaching Conference Interpretation. Udine: Campanotto Editore, 217-218.

Knapp, M.L. (1988). La comunicación no verbal. El cuerpo y el entorno. Barcelona: Paidós comunicación.

Kramer, E. (I963). «Judgement of personal characteristics and emotions from nonverbal properties of speech", Psychological Bulletin 60 (4):408-420.

Mahl, G.F., Schulze, G. (1982). «Die Klassifikation extralinguistischer Phänomenc». En: Scherer, K.R. (ed.). Vokale Kommunikation: nonverbale Aspekte des Spracbverbaltens. Weinheim-Basel: Beitz, 94-104.

Mehrabian, A., Williams, M. (1969). “Nonverbal concomitants of perceived and intended persuasiveness", Journal of Personality and Social Psychology ${ }_{13}(\mathrm{I}): 37-58$.

Navarro Tomás, 'T. (1974). Manual de entonación espanola. Madrid: Guadarrama.

Pöchhacker, F. (I994). Simultandolmetschen als komplexes Handeln. Tubinga: Narr.

Scherer, K.R. (1995). "Expression of emotion in

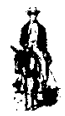


voice and musicm, Journal of Voice $9(3): 235-248$. Shlesinger, M. (1994). «Intonation in the production and perception of simultaneous interpretation». En: Lambert, S. \& Moser-Mercer, B. (eds.). Bridging the Gap. Empirical Research in Simulta- neous Interpretation. Amsterdam/Filadelfia: John Benjamins, 225-236.

Siegel, S.; Castellán, N.J. (1988). Nonparametric Statistics for the Behavioral Sciencies. Singapur: Mc Graw-Hill. 